\title{
THE ACCURACY OF DIGITAL VERSUS CONVENTIONAL OPEN TRAY IMPLANT IMPRESSION TECHNIQUE, IN PARTIALLY EDENTULOUS PATIENTS
}

\author{
Mohamed Farouk Abdalla* and Mohamed M. Dohiem**
}

\begin{abstract}
Abstract: Impression techniques using digital technology seemed to be studied with the accuracy planning to initiate acceptable prosthetic results in implant dentistry. In order to overcome these misfit, digital impression by means of an intra-oral scanner may assist. The purpose of this study was conducted to assess the efficacy of using digital impression technique compared to conventional technique
\end{abstract}

Material and Methods: 8 patients were examined with a total of 22 implants placed, each patient had undergone two impression techniques: a digital impression: intraoral scan body, and a conventional impression: open tray impression, the total deviation between the two impression techniques was measured. This was done by scanning the scan body in patient mouth in group I and digitalizing the conventional open tray impression by scanning the scan body on the cast produced by the open tray in group 2. The scan body was replaced on the software (Exocad software) with custom made abutment and the deviation between the two groups was measured using (Geomagic Control X; 3D systems) GOM inspection software

Results: Total deviation between groups, the open tray conventional impression was compared to hypothesized reference digital impression and were calculated using GOM inspect software. The total deviation was statistically significantly different among the studied groups.

Conclusions: In partially edentulous patients, intraoral oral scanning using intra oral scan bodies improves over all accuracy, than conventional open tray impression technique.

KEYWORDS: Accuracy, Implants, Digital impressions, Partially edentulous, Scan body

\section{INTRODUCTION}

Nowadays with the advent of CAD-CAM technology, prosthetically driven implant placement is promising, by using a digital workflow which can be either direct or indirect approach. The indirect workflow involves making a conventional implant impression which is then digitized in the laboratory by using an optical benchtop scanner and laboratory

* PhD Associate Professor, Prosthodontics Department, Faculty of Dentistry, Cairo University, Cairo Egypt.

** Prosthodontic Department, Faculty of Oral and Dental Medicine, El Zagagig University, Sharqia. 
scan bodies. However, the direct workflow, includes using intraoral scan bodies (ISBs) with an intraoral scanning device which creates a digital scan directly from the patient's mouth. ${ }^{1}$

In conventional impression techniques, if only 1 area is incorrect, the clinician must remake the impression. But, with an intraoral scanner (IOS), if the scan is not performed properly in certain areas, pictures can be easily added without any remakes. Therefore, the intraoral scanning procedure would greatly help those patients who have difficulty with the conventional process of prosthetic fabrication as gag reflex or allergic reaction towards the impression material with advantages that include patient acceptance and the storage and reuse of the scanned data. ${ }^{2,3}$

A digital workflow requires neither conventional plaster nor silicone impressions materials for the manufacturing process of the prosthesis. Consequently, dimensional changes in the physical properties are reduced, and the digital workflow should provide expected permanent dimensional stability. Moreover, faulty impression of conventional technique no more exists, as additive scan and stitching the images with additional scanning through optical impression can be easily achieved allowing the clinician to recognize any deficiencies. ${ }^{4}$

A digital scan is more cost-effective, timeefficient, and easier to adopt than conventional impressions, particularly for inexperienced dentists. ${ }^{5}$

Regarding ISBs, they are implant abutments with varying digitally detectable surface geometry, as long as they are compatible with the designated implant system, IOS and scanner software, the ISB surface is recreated digitally, it then must be exported as a usable file usually in the form of a standard tessellation language (STL) file. A bestfit algorithm is most commonly used to align the CAD geometry with the acquired surface geometry from ISB scanning, this accordingly minimizes the 3dimensional distances between the acquired ISB point cloud (from scanning) and its corresponding reference ISB stored in the implant library. This best fit algorithm drasticly diminishes the root-meansquare errors to below $0.010 \mathrm{~mm}$ which is rated as excellent, whereas values above $0.050 \mathrm{~mm}$ indicate poor correspondence. ${ }^{1}$

Technically speaking, the more point cloud density generated (the more scan points recorded) during scanning, the more precise the virtual surface geometry reconstruction. The opposite is correct as well, in that, absent data points in a point cloud will lead to difficulties when the surface geometry is reconstructed, which might cause multiple errors when attempting to register the image and associate the ISB surface with the implant library which means true image matching is impossible. ${ }^{1,6}$

IOS systems are known to be open, closed, and semi-closed architectures, which is an indication of the degree of flexibility in importing and exporting digital files. Many authors have recommended open or semi-closed systems as they offer the laboratory and clinician more freedom and flexibility. ${ }^{1}$

However, during scanning some reconstructed digital data may be lost, especially when images are stitched together at overlapping areas. When this happens additional chair side adjustments are required. ${ }^{7}$

In other words, the further was the scan from the original situation, the more was the deviation. This outcome is in agreement with former studies approving the reduced trueness of digital implant scans as a result of accumulated errors during image stitching. Such errors usually occur during scanning of long edentulous areas such as fully edentulous arches and free end saddle cases, as there are not enough reference points to help in correct image stitching ${ }^{7}$

No data are available concerning the accuracy of digital scanning versus conventional impressions particularly for partially edentulous arches. Therefore, the primary purpose of this in vivo study was to compare the accuracy of digital 
implant impression by IOS (Medit i500) with the conventional open tray (splinted) impression. The null hypothesis was that splinted open-tray impression has similar accuracy to digital scans for partially edentulous free end saddle arches.

\section{MATERIAL AND METHODS}

This study was a comparative study using conebeam computed tomography (CBCT) imaging and flapless surgical technique to place two mandibular implants under approval of Medical Ethical Committee of Future University; FUE-REC (7)/22021. Patients were selected and diagnosed by (CBCT) and enrolled in the trial from the outpatient clinic of faculty of Dentistry, Future University. 8 male patients age ranging from 30-50, partially edentulous patients (free end saddle situations), were installed with 22 implants: Implant planning \& placement: Cone beam Computed Topography of the patients were taken by $\mathrm{x}$ - ray machine (The Vatech PaX-i Green CT panoramic plus cone beam system delivers $15 \times 15 \mathrm{~cm}$ large field of view cone beam) to create a DICOM file of the patient. Intraoral scanning of the working arch, the opposing arch \& bite registration using Medit i500 to create STL Files of the patient arches.

Digital setting of missing teeth followed by digital articulator were done using Exocad software to accomplish prosthetically driven implant planning. Super imposition of the patient CBCT \& STL using designing software (Real guide software). The surgical guides were created and the implants (Neobiotech) were virtually placed according to the designed prosthesis. After that all the surgeries were done in the mandible using surgical guides with copious irrigation. After 4 months the second stage surgeries were done and healing abutments were placed and left for 2 weeks.

Each patient had undergone two impression techniques before restoration construction including; Conventional impression (Splinted open tray impression technique);

A cast was obtained from a preliminary closed tray impression technique, the short impression transfer copings were unscrewed, and the long transfer copings for the open tray impression were screwed. A verification jig was done to splint the two copings using dental floss and Dura lay acrylic, the jig was sectioned into two pieces to decrease polymerization shrinkage and not to affect the copings position. A custom tray was made on the cast using chemical cured resin (Acrostone) with opened holes corresponding with the two copings. The long impression transfer copings with the jig were screwed in the patient mouth over the implant. The two jig parts were joined together in the patient mouth with Dura lay acrylic. A one step impression technique using putty and light rubber base consistencies (polyvinyl siloxane, Zhermack) were taken with the custom tray after applying tray adhesive 10 minutes prior to the impression. The impression was poured into low expansion type IV dental stone (Zhermack) after the implant analogue (Neobiotech) was repositioned on the coping \& a gingival replica was injected in the impression, around the analogues neck. as seen in Figure 1.

To digitize the open tray impression technique for future testing, scan bodies were screwed on the analogues of the resultant stone casts, followed by digital scanning using intra oral Medit i500 intraoral scanner), as seen in Figure 2.

Digital impression (intraoral scanning using scan bodies):

Scan bodies compatible with the implants placed were screwed manually onto the implants intraorally, and then scanned with intra oral scanner. (Medit i500 intraoral scanner) producing a digital representation of the scan bodies and surrounding structures on the software.

During all scanning procedures, the scanning proceeded in continuous mode around scan bodies, and then again, a detailed scan of the scan bodies was done in circular movement to capture all the details. 
The scans were done by experienced operators in around 5 minutes for each scan then the scans were exported as open- format STL files.

Using the designing software (Exocad Dental CAD) compatible scan bodies were chosen from the software library and superimposed on the scan bodies. As seen in (Figure 3) which enables determination of exact implant position and the implants were added using digital library.

Custom abutments were fabricated on the implant replica of the two groups, conventional impression group (which was digitized) and digital impression group, as seen in (Figure 4)

These custom abutments could be compared in deviations separately with each other without comparing the whole cast or any irrelevant regions, which will generate more accurate results. To increase the accuracy during superimposition the two custom abutments in each scan technique were joined together in one STL file using designing software (Blender software).

A comparison was done between different data acquisition techniques (digital and conventional impressions):

The custom abutment generated by the digital impression was set as the reference, on which all the comparisons will be tested, using GOM (Geomagic inspect X; 3D systems) inspection software. The digitized STL of the conventional open tray impressions was imported as mesh. Then pre-alignment registration method was used, as seen in figure 5.

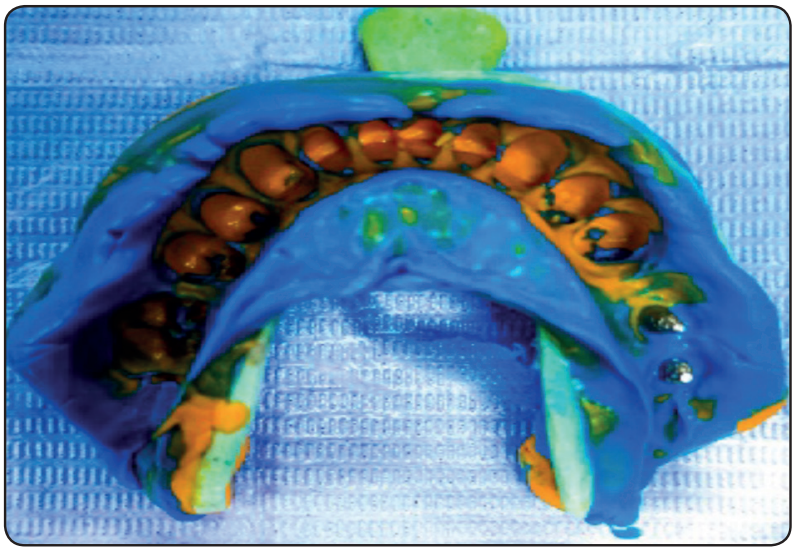

Fig. (1) Open tray impression technique

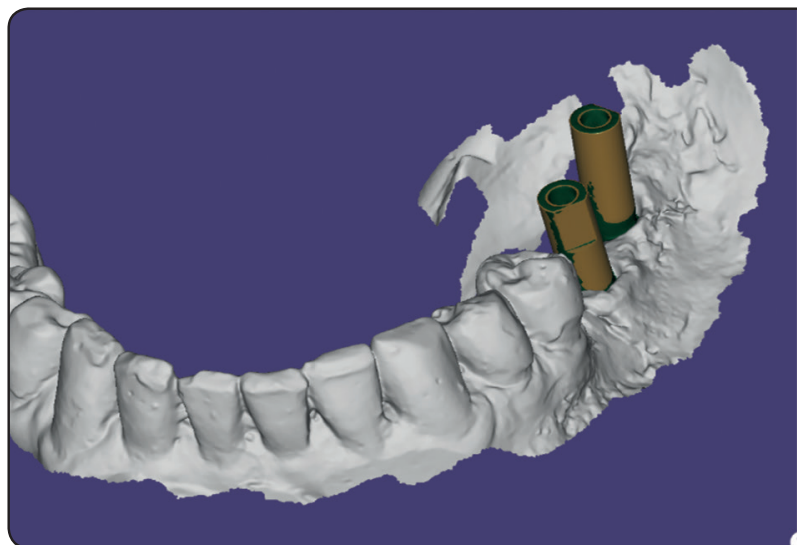

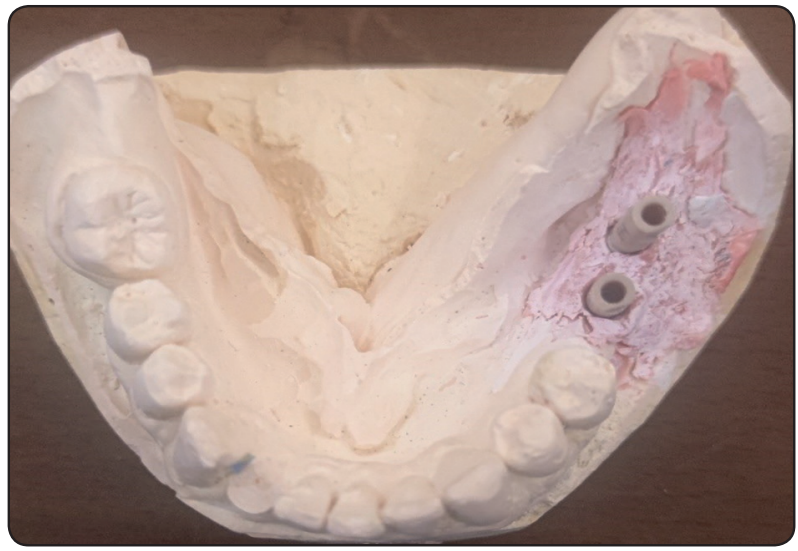

Fig. (2) Scan body loaded on Open tray cast.

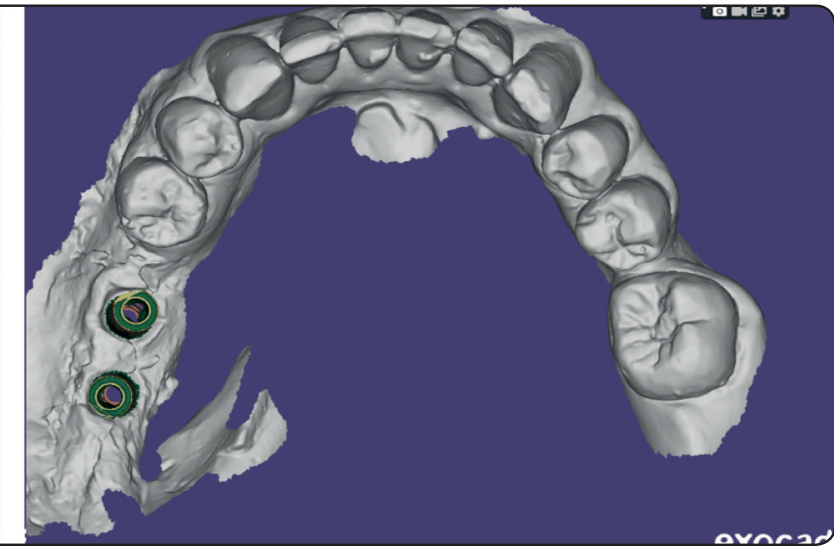

Fig. (3) Registration of scan body from software digital library on cast. 


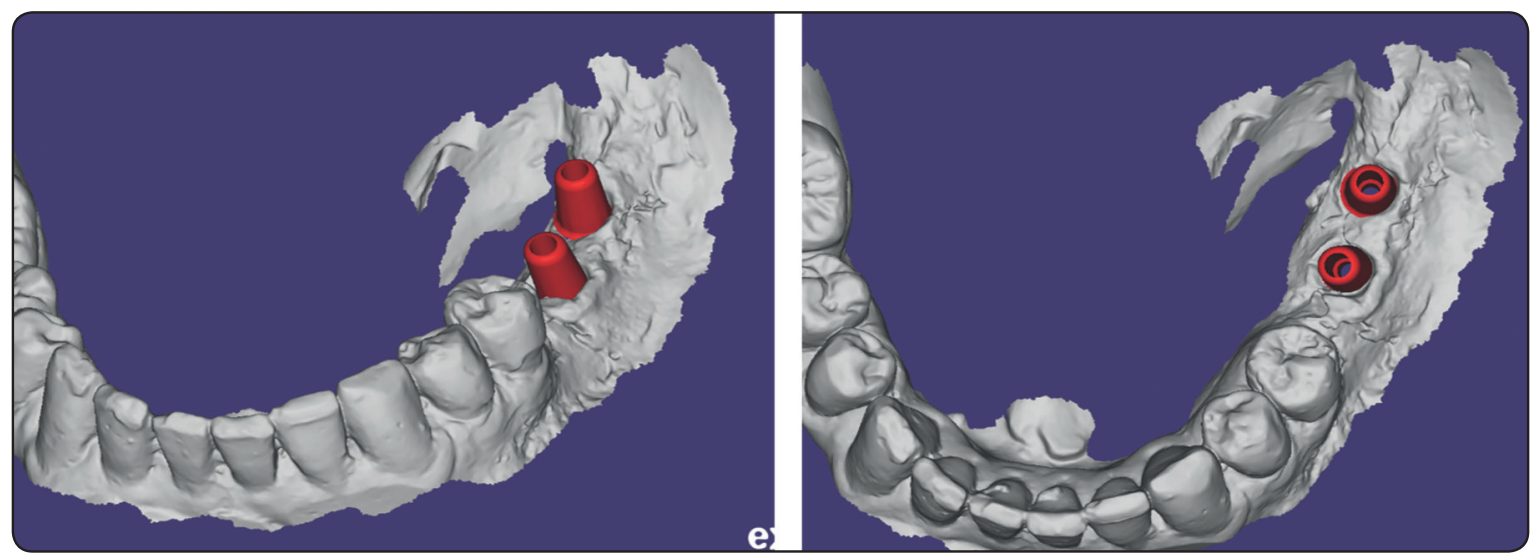

Fig. (4) Virtual designing of custom abutment in the scan body position

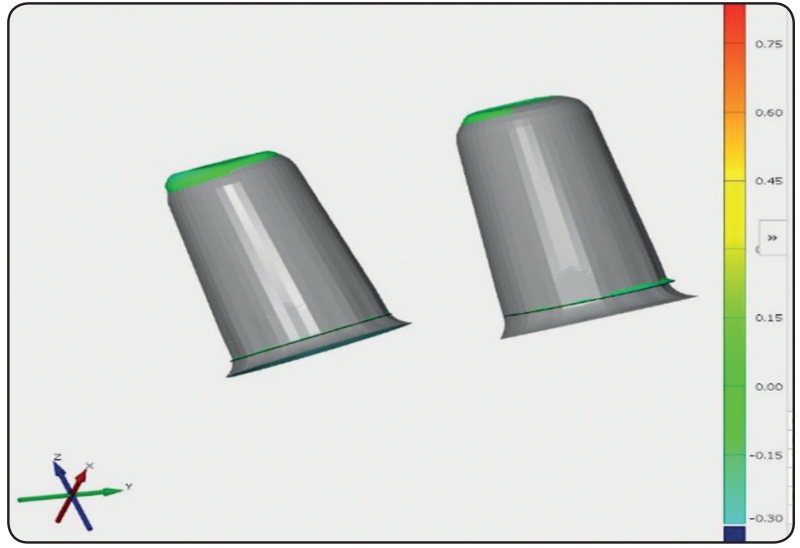

Fig. (5) Scan body comparison: Open tray cast scan body to intra oral scan body

\section{Statistical methodology}

Data were collected and entered to the computer using SPSS (Statistical Package for Social Science) program for statistical analysis (ver 21). Kolmogorov-Smirnov test of normality revealed no significance in the distribution of the variables, so the parametric statistics was adopted. Data were described using minimum, maximum, mean, standard deviation and $95 \%$ confidence interval (CI) of the mean. Comparisons were carried out between two studied independent normally distributed variables using independent sample t-test.

\section{RESULTS}

In the present study, the results in table 1 demonstrated the total deviation in which the digital impression, the conventional impression, and digital impression custom abutment (hypothesized as reference and gold standard) were compared. These deviations were calculated using GOM inspect software and clarified that the total deviation in the digital impression the mean value was " $0.1625 \pm 0.0327 \mathrm{~mm}$ " and $95 \%$ confidence interval (CI) of the mean of " $0.1351-0.1898 \mathrm{~mm}$ ", while in the conventional impression the mean value was " $0.1461 \pm 0.0310 \mathrm{~mm}$ " and $95 \% \mathrm{CI}$ of the mean of “0.1201-0.1720 mm”

The total deviation was statistically significantly different among the two studied groups $\left(\mathrm{t}_{(\mathrm{df}=7)}=\right.$ $14.052, \boldsymbol{p}=<.001 *)$ ). (Table 1)

TABLE (1): Deviation results between digital and conventional impressions.

\begin{tabular}{|c|c|}
\hline Deviation $(\mathbf{m m})$ & Open tray \\
\hline $\mathrm{n}$ & 8 \\
\hline - $\quad$ Min-Max & $0.1134-0.2231$ \\
\hline Mean \pm S.D. & $0.1625 \pm 0.0327$ \\
\hline - $\quad 95 \%$ CI for mean & $0.1351-0.1898$ \\
\hline $\begin{array}{c}\text { One Sample t test } \\
(\text { against hypothesized deviation }=0 \text { ) }\end{array}$ & $\begin{array}{c}\mathrm{t}_{(\mathrm{df}=7)}=14.052 \\
p=<.001 *\end{array}$ \\
\hline
\end{tabular}

$n:$ Number of patients

Min-Max: Minimum - Maximum

S.D.: standard Deviation CI: Confidence interval

* : Statistically significant $(p<0.05)$ 


\section{DISCUSSION}

Passive fit is an important goal for any implantsupported prosthesis, in order to achieve such passivity an accurate impression free from distortion is crucial.

Balouch et al also compared the discrepancy in accuracy between open tray and closed tray impression techniques in $15^{\circ}$ angled implants and concluded that less dimensional changes occurred with the closed tray impression technique. Another study examined the effect of connection type, impression techniques, and positioning angle of the implant on the impression precision. Based on their results, there was no difference in terms of impression exactness, using open and closed tray techniques. ${ }^{11,12}$

The one step polyvinyl siloxane impression technique using putty and light rubber base in a custom tray was used in this study for recording the open tray impression technique. As recommended in a recent study that polyvinyl siloxane impressions obtained using custom made closed tray technique showed superior accuracy and statistically significant difference $(\mathrm{p}<0.01)$ when compared with polyether impressions of non-parallel implants in a partially edentulous arch. ${ }^{13}$

According to a recent systematic review the digital scans are a clinically satisfactory substitute for conventional impression techniques when constructing tooth-borne and implant-supported restorations, especially in short span situations. ${ }^{8}$

Introducing intraoral optical scanners (IOSs) to implant prosthodontics has many benefits, including improved patient comfort and acceptance, dismissal of tray selection; decreased hazard of alteration and distortion during impression making or on removal from patient mouth, stone pouring, disinfecting the impression, and delivery to the dental laboratory besides no cast deformations. Furthermore, the digital scans can be electronically communicated and stored as digital information, increasing efficiency, and reducing costs. ${ }^{1,9}$ Therefore, an optical impression should produce smaller errors with respect to the deviation between implants than a conventional impression would produce. ${ }^{4}$

The results of our study emphasize these findings as the mean deviations of the open tray impression group was " $0.1625 \pm 0.0327$ “ which was statistically significant.

Regarding the discrepancies between the conventional and digital impression this may be attributed to the successive steps that were performed from beginning of the impression till the end of prosthesis construction. ${ }^{15-22}$

These successive steps include, impression making stages, master cast, resin verification jig, waxing, investing, casting, veneer addition and finishing, resulting in a distorted the final outcome..$^{10}$

\section{CONCLUSIONS}

Intraoral scanning using intraoral scan bodies have shown higher accuracy levels than, conventional open tray impression technique, producing a more passive restoration.

\section{REFERENCES}

1. Mizumoto RM, Yilmaz B. Intraoral scan bodies in implant dentistry: A systematic review. J Prosthet Dent. 2018;120(3):343-352. doi:10.1016/j.prosdent.2017.10.029

2. Kim JE, Amelya A, Shin Y, Shim JS. Accuracy of intraoral digital impressions using an artificial landmark. J Prosthet Dent. 2017;117(6):755-761. doi:10.1016/j. prosdent.2016.09.016

3. Cho WT, Bae E Bin, Ahn JJ, Huh JB. Cordless digital workflow for scanning implant-supported prostheses at the abutment level: A dental technique. J Prosthet Dent. 2020;124(4):428-430. doi:10.1016/j.prosdent.2019.11.003

4. Ajioka H, Kihara H, Odaira C, Kobayashi T, Kondo H. Examination of the position accuracy of implant abutments reproduced by intra-oral optical impression. PLoS One. 2016;11(10):1-12. doi:10.1371/journal.pone.0164048 
5. Li J, Chen Z, Wang M, Wang HL, Yu H. Dynamic changes of peri-implant soft tissue after interim restoration removal during a digital intraoral scan. J Prosthet Dent. 2019;122(3):288-294. doi:10.1016/j.prosdent.2018.07.020

6. Park S, Choi Y, Lee D. The effect of the improperly scanned scan body images on the accuracy of virtual implant positioning in computer-aided design software. 2020:107-113.

7. Kim RJY, Benic GI, Park JM. Trueness of ten intraoral scanners in determining the positions of simulated implant scan bodies. Sci Rep. 2021;11(1):1-9. doi:10.1038/s41598021-82218-z

8. Mizumoto RM, Yilmaz B, McGlumphy EA, Seidt J, Johnston WM. Accuracy of different digital scanning techniques and scan bodies for complete-arch implantsupported prostheses. J Prosthet Dent. 2020;123(1):96104. doi:10.1016/j.prosdent.2019.01.003

9. Marghalani A, Weber HP, Finkelman M, Kudara Y, El Rafie K, Papaspyridakos P. Digital versus conventional implant impressions for partially edentulous arches: An evaluation of accuracy. J Prosthet Dent. 2018;119(4):574579. doi:10.1016/j.prosdent.2017.07.002

10. A. G. Wee, S. A. Aquilino and RLS. Strategies to achieve fit in implant prosthodontics: a review of the literature. Int J Prosthodont. 1999;12:167-178.

11. Balouch F, Jalalian E, Nikkheslat M, et al. Comparison of Dimensional Accuracy between Open-Tray and ClosedTray Implant Impression Technique in $15^{\circ}$ Angled Implants. J Dent (Shiraz, Iran). 2013;14(3):96-102.

12. Heidari B, Allahbakhshi H, Services H. Comparative Study of Dimensional Accuracy in Three Dental Implant Impression Techniques : Open Tray, Closed Tray with Impression Coping, and Snap Cap. 2018;(August). doi:10.5005/jp-journals-10024-2368

13. Parameshwari G, Chittaranjan B, Sudhir Chary N, Anulekha Avinash CK, Taruna M, Ramureddy M. Evaluation of accuracy of various impression techniques and impression materials in recording multiple implants placed unilaterally in a partially edentulous mandible- An in vitro study. J Clin Exp Dent. 2018;10(4):e388-e395. doi: $10.4317 /$ jced.54726

14. Imen D. Open Tray Impression Technique Using the Direct Pick-Up Coping: A Case Report. Mod Approaches Dent Oral Heal Care. 2018;3(1):233-236. doi:10.32474/ madohc.2018.03.000155

15. Moreira AHJ, Rodrigues NF, Pinho ACM, Fonseca JC, Vilaça JL. Accuracy comparison of implant impression techniques: a systematic review. Clin Implant Dent Relat Res 2015;17(suppl 2):e751-64.

16. Mizumoto RM, Yilmaz B, McGlumphy EA, Seidt J, Johnston WM. The accuracy of different digital scanning techniques and scan bodies for complete-arch implantsupported prostheses. J Prosthet Dent 2018;120(3):343-52.

17. Giménez B, Pradíes G, Martínez-Rus F, Özcan M. Accuracy of two digital implant impression systems based on confocal microscopy with variations in customized software and clinical parameters. Int J Oral Maxillofac Implants 2015;30:56-64.

18. Abduo J, Elseyoufi M: Accuracy of intraoral scanners: a systematic review of influencing factors. Eur J Prosthodont Restor Dent 2018;26:101-121

19. Basaki K, Alkumru H, De Souza G, et al: Accuracy of digital vs conventional implant impression approach: a three-dimensional comparative in vitro analysis. Int J Oral Maxillofac Implants 2017;32:792-799

20. Moura RV, Kojima AN, Saraceni CHC, et al: Evaluation of the accuracy of conventional and digital impression techniques for implant restorations. J Prosthodont 2019;28:e530-e535

21. Flugge TV, Att W, Metzger MC, et al: Precision of dental implant digitization using intraoral scanners. Int J Prosthodont 2016;29:277-283

22. Alsharbaty MHM, Alikhasi M, Zarrati S, et al: A clinical comparative study of 3-dimensional accuracy between digital and conventional implant impression techniques. J Prosthodont 2019;28:e902-e908 